\title{
Physical Quanta in Quasicrystal Diffraction
}

\author{
Antony J. Bourdillon \\ UHRL, San Jose, CA, USA \\ Email: bourdillona@sbcglobal.net
}

How to cite this paper: Bourdillon, A.J. (2021) Physical Quanta in Quasicrystal Diffraction. Journal of Modern Physics, 12, 16181632.

https://doi.org/10.4236/jmp.2021.1212096

Received: August 29, 2021

Accepted: October 16, 2021

Published: October 19, 2021

Copyright $\odot 2021$ by author(s) and Scientific Research Publishing Inc. This work is licensed under the Creative Commons Attribution International License (CC BY 4.0).

http://creativecommons.org/licenses/by/4.0/

\begin{abstract}
Diffraction in quasicrystals is in irrational and geometric series with icosahedral point group symmetry. None of these features are allowed in Bragg diffraction, so a special theory is required. By means of a hierarchic model, the present work displays exact agreement between an analytic metric, with a numeric description of diffraction in quasicrystals-one that is founded on quasi-structure-factors that are completely indexed in 3-dimensions. At the quasi-Bragg condition, the steady state wave function of incident radiation is used to show how resonant response, in metrical space and time, enables coherent interaction between the periodic wave packet and hierarchic quasicrystal. The quasi-Bloch wave is invariant about all translations $a \tau^{m}$, where $a$ is the quasi-lattice parameter. This is numerically derived, analyzed, measured, verified and complete. The hierarchic model is mapped in reverse density contrast, and matches the pattern and dimensions of phase-contrast, optimum-defocus images. Four tiers in the hierarchy of icosahedra are confirmed, along with randomization of higher order patterns when the specimen foil is oriented only degrees off the horizontal. This explains why images have been falsely described as having "no translational symmetry".
\end{abstract}

\section{Keywords}

Quasicrystal, Icosahedra, Hierarchic, Resonant Response, Harmonic, Irrational, Geometric Series, Metric, Diffraction Quanta

\section{Introduction}

The most profound physical effect that is found in a quasicrystal (QC), is diffraction in geometric series. The effect is incompatible with Bragg's law for crystals, which is in integral order $n$. His ordering is due to the physical harmonies that occur at a Bragg condition, between a crystal scattering a periodic probewhether of photons or electrons-from planes of atoms that are ordered and periodic. 
By contrast, the QC was described as a "Metallic phase with long range order and no translational symmetry" [1]. Evidence for long range order is implied by its sharp diffraction. However, there is imaging evidence for hierarchic symmetry, at least in reasonably short range, and this may be called translational. Furthermore, numeric and analytic simulations prove that the probe's quasi-Bloch wave also has, at the quasi-Bragg condition, translational symmetry about $a \tau^{m}$, where $a$ is the measured and calculated quasi-lattice parameter [2]; and where $\tau \equiv(1+\sqrt{5}) / 2$ is the golden section; while the hierarchic order $m$ is integral.

Bragg diffraction is, in momentum space, a quantum effect. It resembles quantized transitions between energy states in the hydrogen atom: we know these states are harmonic, in time and space, because they are solutions to Schrödinger's equation. In this paper, we refresh the argument and add new data, i.e. by projecting atoms in the Hierarchical Icosahedral (HI) structure onto the 5-fold (1 $\tau 0)$ plane. This is done in reverse contrast for the first time and compares closely with transmission electron microscope (TEM) images, including randomization of off-plane cells in the long range.

\section{Harmonic Scattering between a Periodic Probe and Hierarchic Quasicrystal}

In crystals, elastic scattering is illustrated in Figure 1. Applying Bragg's law, quanta $n \Delta k$ may be generated from a quantum $\Delta k=\hbar \cos (\theta) / \lambda$ where the order $n$ is integral; $\theta$ is the Bragg angle; $\lambda$ the wavelength of the scattered radiation, and $\hbar$ the reduced Planck constant. In case of small angles, as in high energy electron scattering, the interplanar spacing on first order $d_{n=1} \simeq \lambda / 2 \theta$ is unique and harmonic at any Bragg order $n$, so that the scattering crystal diffraction is periodic-like the crystal-and harmonic.

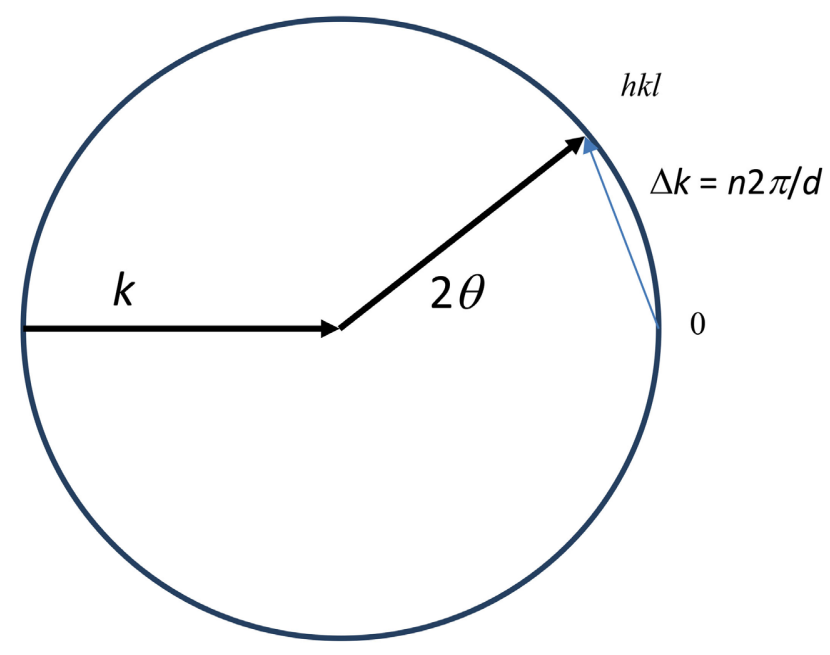

Figure 1. Following Bragg's law, quanta of momentum $n \Delta k$ are transferred for each scattered beam on the periodic Bragg diffraction pattern: the periodic probe and crystal lattice cooperatively harmonize in the well-known way. In high energy electron scattering at the quasi-Bragg condition, the irrational quasi-lattice that is hierarchic and geometric, will be found to cooperate with the beam probe via a quasi-Bloch wave and metric. 
The physical basis for this quantization is similar for the quantized eigenvalues of Schrödinger's hydrogen atom in energy space: the eigenstates do not self destruct by interference precisely because their orbits are harmonic as in Bohr's atom. In electron state transitions, energy is transported in wavepackets with $\Delta E=R_{H}\left(n_{f}^{-2}-n_{i}^{-2}\right)$, where $n_{i}$ and $n_{f}$ are initial and final state principal quantum numbers, and $R_{H}$ is the Rydberg unit of energy.

The same theoretic basis is needed for diffraction in QCs that have multiple interplanar spacings, that are not periodic, are not harmonic and that-far from being integral—are actually irrational and in geometric series, $\tau^{m}$; with $m=-\infty,-1,0,1,2,3, \cdots$. The quasi-Bragg law is observed [3] [4]:

$$
\tau^{m} \lambda=2 d^{\prime} \sin \left(\theta^{\prime}\right)
$$

where the quasi-Bragg angle turns out to be $\theta^{\prime}=\theta / c_{s}$; with $c_{s}$ the coherence factor, or metric [5] that will be defined below; and where the quasi-interplanarspacing is found to be $d^{\prime}=d c_{s}$. Every atom scatters from locations on jumbled planes with multiple values of $d$. We shall see how coherent scattering is won in the QC from this group of properties: the metric, that results from resonant response of the periodic probe to the hierarchic quasi-lattice [6], digitizes its irrational measure, and harmonizes the probe with the QC. The hierarchy is uniquely icosahedral, with cells, clusters and superclusters uniformly aligned by multiple edge sharing at each element.

Quantum mathematics is not enough: to understand digitization and harmony in the scattered wave, we need to briefly recall radiant scatterers in the broader scope of physics. After the Michelson-Morley experiment falsified the ether hypothesis, an attempt was made to salvage it with the Lorentz transformation. This was not as successful as Einstein's foundational relativity: "Physical laws are invariant in all inertial reference frames." A consequence is the Pythagorean equation: $E^{2}=\boldsymbol{p}^{2} c^{2}+m_{0}^{2} c^{4}$. After quantization by Planck's law for energy $E$; and by the de Broglie hypothesis for momentum $p$; and with simplification of units $\hbar$ $=c=1$ for the reduced Planck constant $\hbar$; and for the speed of light $c$, the rest mass reduces to:

$$
m_{0}^{2}=\omega^{2}-k^{2}=(\omega+k)(\omega-k) .
$$

The brackets govern in turn particulate conservation laws, and response that is wave-like. The former bracket is real; the latter imaginary. In the diffractive interactions considered here, the response is resonant and harmonic. The particle-wave duality is thus formulated in respective real and imaginary parts of the normal wave packet [7]:

$$
\varphi=A \cdot \exp \left(\frac{X^{2}}{2 \sigma^{2}}+X\right)
$$

with imaginary:

$$
X=i(\bar{\omega} t-\bar{k} x)
$$

where $\sigma$ depends on initial conditions that determine the coherence of the pack- 
et in space and time ${ }^{1}\left(\right.$ rank $\left.R^{4}\right)$, and where $A^{2}$ is a normalizing constant ${ }^{2}$. The angular frequencies $\omega$ and wave vectors $k$ are in fact distributed, but they are represented in Equation (1) by mean values. The intensity $\phi^{*} \phi$ is a probability density function for a particle, or for a photon having zero mass, $m_{0}=0$. Notice that the response is elastic because its absolute, measurable value is unity: $\left(\mathrm{e}^{X}\right)^{*} \mathrm{e}^{X}=1$, everywhere and at all time.

When an electron binds to an atom, the latter's central potential wraps the extended, interacting electron wavepacket into compact harmonic space. This process would be destructive if the wavefunction orbitals were not harmonic in motion, with discreet wavevector and frequency. In quantized quasicrystal diffraction, the scattering is likewise integrated over space and time. Every atom scatters: the integration occurs over multiple, jumbled planes. Scattering at the quasi-Bragg angle is due to discreet integrands and these will be calculated in the next section by the quasi-structure factor (QSF). This factor is independent of scattering angle which is theoretically unknown a priori. The QSF is therefore descriptive where Bragg's law is not. However, we will show how the scattering angle is calculated numerically, and exactly matched analytically.

Meanwhile, Equation (2) represents the steady state for the incident radiation and, after a transition involving a change in wave-vector $k$, it will represent likewise, the steady state of the diffracted wave. When the incident wave strikes the QC, it interacts with its electric field to form quasi-Bloch waves. You can think of these as lattice images observed in crystalline thin foils in the two-beam condition. The waves, as they proceed through the QC, oscillate (by the pendellösung effect) between the two beams (in crystals: [8]; cf. in QCs: [2] [9]). In wedge specimens, this oscillation produces images of "thickness fringes". The process requires and ensures harmonic interaction, in both space and time, in the propagation direction as in the transverse. An example will be given in the next section, though the "quasi-lattice image" will not be a true lattice image because of the metric.

Notice that Equations (3) effectively linearize the second order Equation (2) of special relativity, and so do for the free particle what Dirac's equation does for the bound electronic states in atoms. Moreover, the Equations (2) separate the propagation direction from the transverse direction, and this has many consequences including: solutions for negative mass [10] $]^{3}$, phase velocity [7], uncertainty, Newton's second law, electron spin (as induced paramagnetism in phase space, that is consistent with Hundt's rules in atomic structure), intrinsic magnetic radius [11] and fine structure constant, reduction of the wave packet [12] etc. The equations apply in harmonious diffraction by quasicrystals and crystals, as they do in the Schrödinger equation that operates on steady-state, harmonic ${ }^{1}$ Typically, the coherence has transverse components, $\sigma_{y}, \sigma_{z}$ as well, but these are only implied here for simplicity. Furthermore, when an atom is excited or decays, its central potential wraps the extended, interacting wavepacket into compact harmonic space.

${ }^{2} A^{*} A=\left(\int \exp \left(X^{2} / \sigma^{2}\right) \cdot \mathrm{d} \tau\right)^{-1}$.

${ }^{3}$ To avoid unphysical singularities when $k=-m_{o} \mathcal{C}$, our antiparticle travels with forward velocity but reverse spin (cf. [6]). 
bases. The diffraction orders and quantum numbers respectively describe interaction requirements that are quantized by necessary constructive interference over space and time. The formalism in Equation (3) will enable our understanding of the fundamental interaction required in the coherent diffraction in QCs that will be described in what follows.

It is obvious that the diffraction depends on the phase properties of the probe, especially its wavelength; but it also resonates coherently in both space and time. Meanwhile, the metric provides corresponding coherence in spite of the multitude of interplanar spacings that have precise order and symmetry in the HI, as QSFs will prove. The diffraction pattern of $\mathrm{i}-\mathrm{Al}_{6} \mathrm{Mn}$ has been completely indexed and simulated in three dimensions [4] [13]. Dimensions should not be multiplied without necessity. It is one role of theory to invent short cuts, but quantum math $^{4}$ turned the egg upside down and ate the cup. In Aristotle's informal logic, the fallacy is called, Ignoratio Elenchi, which is translated: "missing the point".

\section{The Metric: Numeric, Analytic and Measured}

Since QCs do not obey Bragg's law of diffraction, nothing is known a priori about corresponding relationships between $\theta^{\prime}, \lambda$ and $d^{\prime}$. However, the structure factor (SF) method is independent of $\theta$. we can use the method by applying the known relationship between $d^{\prime}$ and the index $h_{h k l}$ in cubic structures: $d=a / \sqrt{h^{2}+k^{2}+l^{2}}$. Here $a$ represents the lattice parameter, and subscripts $h$, $k$ and $I$ represent the 3-dimensional indices in the diffraction pattern [14] [15]. It turns out that all structure-factors in the QC are zero. The implied absence of diffraction should be expected in a solid whose images demonstrate multiple interplanar atomic spacings. However it turns out further, that by introducing a coherence factor $c_{s}$, which is specific to the hierarchic icosahedral structure, a quasi-Bragg condition is discovered that is as sharp as the Bragg condition commonly observed by rocking crystals. The coherence factor is discovered by simulations in which the factor is numerically scanned while evaluating the quasi-structure-factor (QSF), first over the unit cell (order $p=0$ ) with atomic scattering factors $f_{i}=f_{\mathrm{Al}}$ or $f_{\mathrm{Mn}}$ in Equation (4), and secondly over clusters order $p$, by iteratively adding cluster centers at $\boldsymbol{r}=\boldsymbol{r}_{c c}$ in Equation (5) [2]:

$$
\begin{gathered}
F_{h k l}=\Sigma_{i}^{\mathrm{Al}, \mathrm{Mn}} f_{i} \cos \left(2 \pi \cdot c_{s}\left(\boldsymbol{h}_{h k l} \cdot \boldsymbol{r}_{i}\right)\right) \\
F_{h k l}^{p}=F_{h k l}^{p-1} \cdot \Sigma_{c c} \cos \left(2 \pi \cdot c_{s} \tau^{2 p}\left(\boldsymbol{h}_{h k l} \cdot \boldsymbol{r}_{c c}\right)\right)
\end{gathered}
$$

All atoms scatter.

In crystals, the SF is simpler and is represented by Equation (4) with $c_{s}=1$. There, the calculation is comparatively easy because the summation is limited to one unit cell which repeats periodically. Symmetry in the unit cell often forces $F_{h k l}=0$, or to a small range of values. In QCs, by contrast, the QSFs are calculated over all the atoms in a selected order of HI. They contain a spectrum of ${ }^{4}$ Including P.A.M. Dirac's The Principles of Quantum Mechanics (1958) Oxford, with such ugly concepts as unstable wavepackets; unexamined internal motion; unphysical electron speed $V=\mathcal{C}$; etc.: anomalies falsified by QC diffraction. 
amplitudes, whose intensities match measured intensities [4].

The main argument in favor of the hierarchic structural solution is-in physics-overwhelming ${ }^{5}$ : uniquely, the solution is in geometric series, fitting the diffraction pattern, and it is consistent with imaging that will be simulated below.

The most substantial result is the discovery of the numeric metric. This will be subsequently proven, firstly by its analytic explanation, and secondly by verified measurement of the lattice parameter. These calculations were initially thought to be ideal; later we will discuss defects, both short range vacancies and interstitials, and also long-range quasi-lattice congruities.

Figure 2 shows one simulation for the $(\tau 00)$ intensity in a supercluster order 6. Here, $c_{s}$ is scanned across the quasi-Bragg condition. There is no Bragg diffraction when $c_{s}=1$; diffraction occurs at the quasi-Bragg condition when $c_{s}=$ 0.894 .

The coherence factor is the same for all of the beams in the original data [1] and so is called a metric. What is it? Equation (4) and (5) show that it has the same influence as the lattice parameter or reciprocal lattice parameter. The coherence factor is a virtual breathing strain that switches the quasi-Bragg diffraction on or off, like the rocking curve of a rotating crystal. In consequence, the quasi-Bragg angle in QCs is increased from the corresponding Bragg angle in crystals by about $11 \%$. This difference will become significant in the measurement of the quasi-lattice parameter.

The most remarkable feature of the QSF is its precise value for $c_{s}$, as calculated in the HI model (Figure 2)-less than $1 / 1000^{\text {th }}$ of the quasi-Bragg angle. After analyzing the metric when it is applied to quasi-Bloch waves, the special translational symmetry will become apparent.

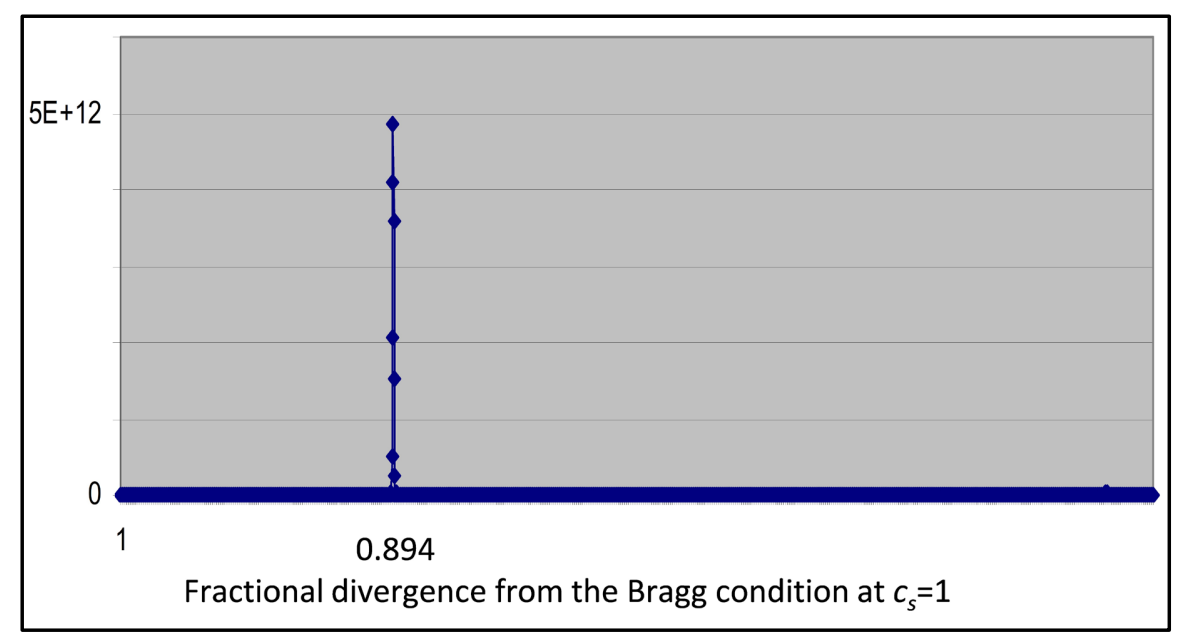

Figure 2. Quasi structure factor for the HI ( 200$)$ diffracted beam, due to a supercluster order 6. On scanning $c_{s}$, the QSF is zero at the Bragg condition when $c_{s}=1$; at the quasi-Bragg condition $c_{s}=0.894$. The quasi-Bragg angle is $\sim 11 \%$ greater than the corresponding Bragg angle in crystals due to equivalent, except periodic, $d$. The calculated line width is less than $1 / 1000^{\text {th }}$ of the corresponding Bragg angle.

${ }^{5}$ In mathematics, all axioms are allowed. 
The coherence factor $c_{s}$ is analyzed as follows. Consider the following mathematical fact that is proved by mathematical induction:

$$
\tau^{m}=F_{m}(1, \tau)=\partial_{(m, 0)}+F_{m+1}(0,1)+F_{m}(0,1) \tau
$$

where $F_{m}(a, b)$ represents the $m^{\text {th }}$ term in the Fibonacci sequence, base $(a, b)$, and $\partial_{(m, 0)}$ is the Dirac delta function. From the terms at right, the natural part is separable by approximating $\tau \rightarrow 3 / 2$, and the metric function is derived by subtracting out the irrational residue from its corresponding geometric order $\tau^{m}$ :

$$
\frac{1}{c_{s}}=1+\frac{\tau^{m}-F_{m+4} / 2}{F_{m+1}}=\frac{1}{0.894}
$$

This function turns out to be the exact inverse of the numeric metric that was derived from the QSF. The fact is extremely surprising because the numeric and analytic derivations are independent. The formula is identical for all terms in the series, and in all three spatial dimensions. In QCs, the divergence from the Bragg angle is due to the irrational parts of the indices; this divergence digitizes the separated natural part that provides coherent, harmonic scattering [2], i.e. with $c_{\mathrm{s}}=1$.

Details of the effects of the metric function are illustrated by quasi-Bloch waves. These can be thought of as amplitudes used to construct quasi-lattice images in the 2-beam condition. The quasi-Bloch waves are created in the incident probe by atomic potentials in the hierarchic icosahedral structure. Consider first, a typical Bloch wave in a crystal, like the blue wave in Figure 3. The wave is commensurate with the unit cell and with all unit cells, periodically repeating. This wave is not commensurate with the irrational and geometric series intercepts, that mark the central locations of atoms, cells, clusters and super clusters, of whatever order. However, when the blue axis is multiplied by the metric function, the resulting red quasi-Bloch wave becomes commensurate. This is partly due to the rational denominator $F_{m+1}$ in Equation (8). The metric function digitizes and harmonizes the periodic probe when it enters the irrational, geometric, quasi-lattice potential. The function enables coherent diffraction when the probe scatters from the many aperiodic atoms in the hierarchic quasi-lattice. The coherence results from the combined and characteristic translations in the HI. This coherence is calculated in the QSFs (Figure 2).

Notice that the probe contains long range order with translational symmetry about all geometric intercepts $a \tau^{m}$, where $a$ is the quasi-lattice parameter. The symmetry occurs also in short range in the quasi-Bloch wave. Only with the analysis provided here, can the parameter be measured.

In QCs, the "lattice parameter" was measured a long time ago, based on the doubtful supposition of Bragg's law of diffraction. The measurement compared the dominant scattering angle in the 5 -fold diffraction pattern of $\mathrm{i}$ - $\mathrm{Al}_{6} \mathrm{Mn}$ with a known scattering angle in a crystalline second phase. The measured value was $0.206 \pm 0.005 \mathrm{~nm}$ [16] [17]. This corresponds to the indexed beam $(\tau, 0,0)$ and corresponds also with the cell length and intercellular spacing throughout the QC (Figure 4), namely $a \tau$, where the golden-rectangle cross-section of the unit cell is $\tau \times 1$ in icosahedral units, or $a(\tau \times 1)$ in SI. 


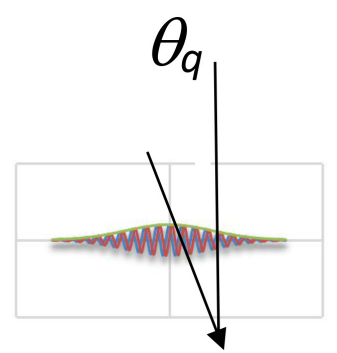

(a)

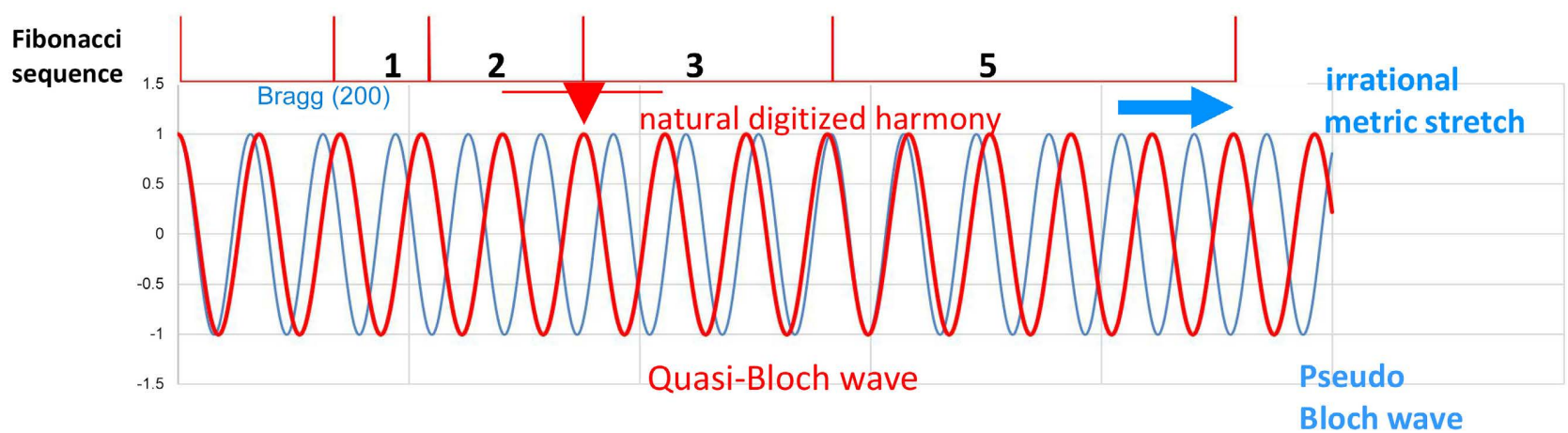

(b)

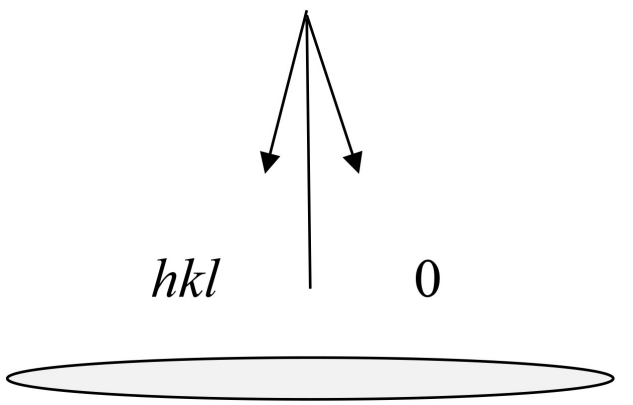

(c)

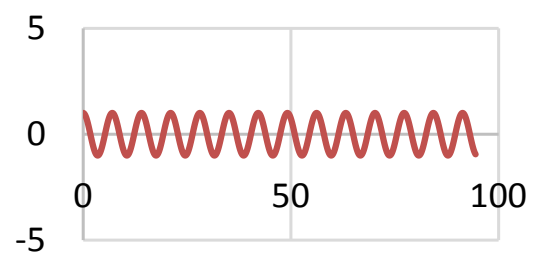

(d)

Figure 3. (a) Incident, time-dependent, beam probe (Equation (2)), rank $R^{4}$, inclined at quasi-Bragg angle from normal: $\theta_{q}=\sin ^{-1}\left(\lambda \sqrt{h^{2}+k^{2}+l^{2}}\right) /\left(2 a c_{s}\right)$, in electron scattering. (b) Crystalline Bloch waves (blue) are commensurate with their corresponding periodic crystal lattice at the Bragg condition. When this wave is stretched horizontally by the inverse coherence factor $1 / c_{s}$, the quasi-Bloch-wave (red) commensurates with the irrational, geometric and hierarchic, quasi-lattice. Its geometric order is represented by the intercepts on the horizontal line above it. The digitized number of periodic cycles between successive intercepts is in Fibonacci sequence (denominator in Equation (3)), and the diffraction is logarithmically periodic. The natural and irrational parts of the indices are separable: the irrational part is expressed by the metric stretch; the natural part scatters with sharp, coherent diffraction. (c) Diffracted beams emitted beneath foil, including indices. (d) In TEM, beams can be magnetically refocused to produce a quasi-lattice image of the probe at the base of the specimen foil. The lattice image is the interference due to the superposition. 


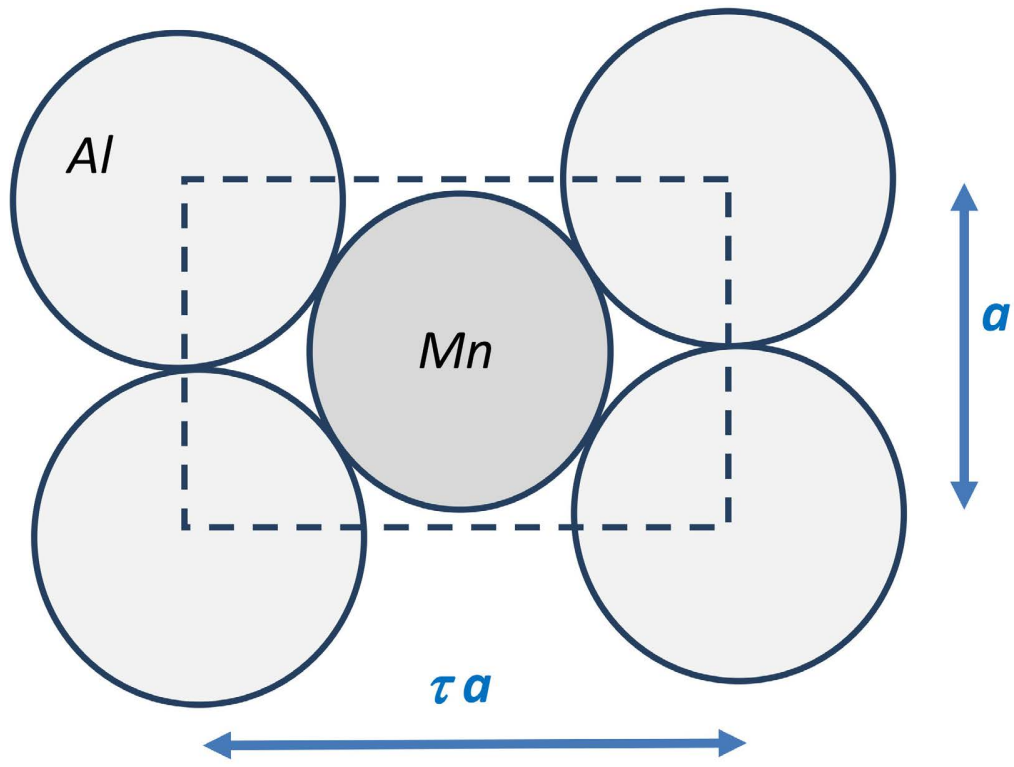

Figure 4. Golden-rectangle cross-section of the icosahedral unit cell in i- $\mathrm{Al}_{6} \mathrm{Mn}$, having side width $a$ (the lattice parameter) and length $\tau a$. The unit cell contains 15 identical sections at various orientations. The structure is extremely dense, and depends on atomic diameter ratios $d_{\mathrm{Mn}} / d_{\mathrm{Al}}=\sqrt{1+\tau^{2}}-1$.

By using the same formula as applies to cubic crystals to find the interplanar quasi-spacing $d=a / \sqrt{h^{2}+k^{2}+l^{2}}$, the correction for the quasi-Bragg law is therefore given $a=0.205 \times c_{s} \tau \pm 0.05 \mathrm{~nm}$. The resulting value is close to the standard value of the diameter of $\mathrm{Al}$ in the pure metal. This measurement confirms the structural model and method.

\section{Ideals and Defects}

The overwhelming advantage of the ideal $\mathrm{HI}$ model is the explanation it provides for the observed geometric series diffraction that is apparently unique to QCs. A subsidiary advantage is the explanation for icosahedral symmetry in the diffraction pattern from a structure that repeats, uniquely, as hierarchic. The repetition occurs at the unit cell level and throughout the quasi-lattice. The unit cell is denser than can occur in crystals; but space-filling is taken up beyond the unit. The cell contains a single $\mathrm{Mn}$ atom at its center, surrounded by 12 closely packed $\mathrm{Al}$ atoms [2]. The extreme density depends on the atomic diameter ratios, $d_{\text {solute }} / d_{\text {solvent }}=\sqrt{1+\tau^{2}}-1$. At short range, space occupation is simple enough and is consistent with phase-contrast, optimum-defocus imaging up to the first order of superclusters [18]. At this range, vacancies at cluster centers and interstitial atoms (including tetrahedra or unit cells) at supercluster centers minimally affect the diffraction pattern [5]. However, extracellular holes become an ever-expanding problem in higher orders. Now that the metric is consistently understood in the ideal HI, we return to less conventional ways in which filling may occur in higher orders of the hierarchy.

In crystals, the SF is calculated in the periodically repeating unit cell. The 
symmetry of a single unit cell defines the diffraction pattern (whether simple cubic, face-centered cubic (fcc), body centered cubic etc.) for the whole crystal, repeating lattice and all. All cells are uniquely aligned because they are face sharing. Meanwhile, no crystal is perfect: interstitials and vacancies are common; as are growth defects such as twinning; and various dislocations that are products of deformation strains. We understand that such defects should be common in $\mathrm{HI}$ as they would relieve quasi-lattice stresses, especially those arising from uneven atomic densities near holes but also due to thermal stresses during rapid cooling.

The HI structure proved essential to demonstrating the effects of irrational indices in setting the metric and in establishing harmonic scattering in diffraction. Notice firstly that cell alignment is necessary for the sharp diffraction pattern. This must be due to the multiple edge sharing of the icosahedral cells, clusters and superclusters as they indeed occur in the HI. Moreover, since, in crystals, the diffraction pattern symmetry is set by the structure factor of the unit cell, we need to assess, for QCs, the relative importance of the unit cells versus quasi-lattice structures, i.e. short range versus long range, as needed to ensure coherence and pattern identity. After describing atomic maps in oriented thin films, we shall consider long range lattice irregularities.

\section{Maps onto the 5-Fold Icosahedral (1 $\tau 0)$ Plane}

The decoration of the unit cell (in icosahedral units) is as follows:

$M n$ on the site $(0,0,0)$, with

$A l$ on the 12 permutations of $( \pm 1, \pm \tau, 0)$.

On the cluster:

12 unit cells centered on the 12 permutations of $\left( \pm \tau, \pm \tau^{2}, 0\right)$.

On the supercluster, order $p$ :

12 superclusters order $(p-1)$ centered on permutations of $\tau^{2 p}\left( \pm \tau, \pm \tau^{2}, 0\right)$.

Our purpose is to simulate the most significant atomic maps that can be observed in the original diatomic quasi-crystal, bearing in mind that our phasecontrast, optimum-defocus shows both limited resolution, and reverse contrast [2] [14] [18]. It is relatively simple to plot every atom: we construct the structure on Cartesian axes and rotate it to make the $(1 \tau 0)$ horizontal [19]. Then we select a foil thickness as observed in TEM. This thickness might contain the hemisphere of a cluster, for example, or a horizontal slice of the structure. The result has so much structure with unaccounted intensity that it hides the desired pattern [5]. We simplify by plotting only to the low resolution that represents unit cells. We do this by omitting the weakly scattering $\mathrm{Al}$ atoms (Figure 5(a)). The resulting pattern shows the diameter of the cluster to be 0.9 $\mathrm{nm}$ which is close enough to Bursill and Peng's measurement $(0.85 \mathrm{~nm})$ based on microscope micron markers and the indefiniteness of their reverse contrast. Our next step is to reverse our simulated contrast by omitting the $\mathrm{Al}$ atoms; instead, simulating a cluster of cluster peripheries (Figure 5(b)). The peripheries 


\section{Supercluster showing $M n$}

\section{atoms above median plane}

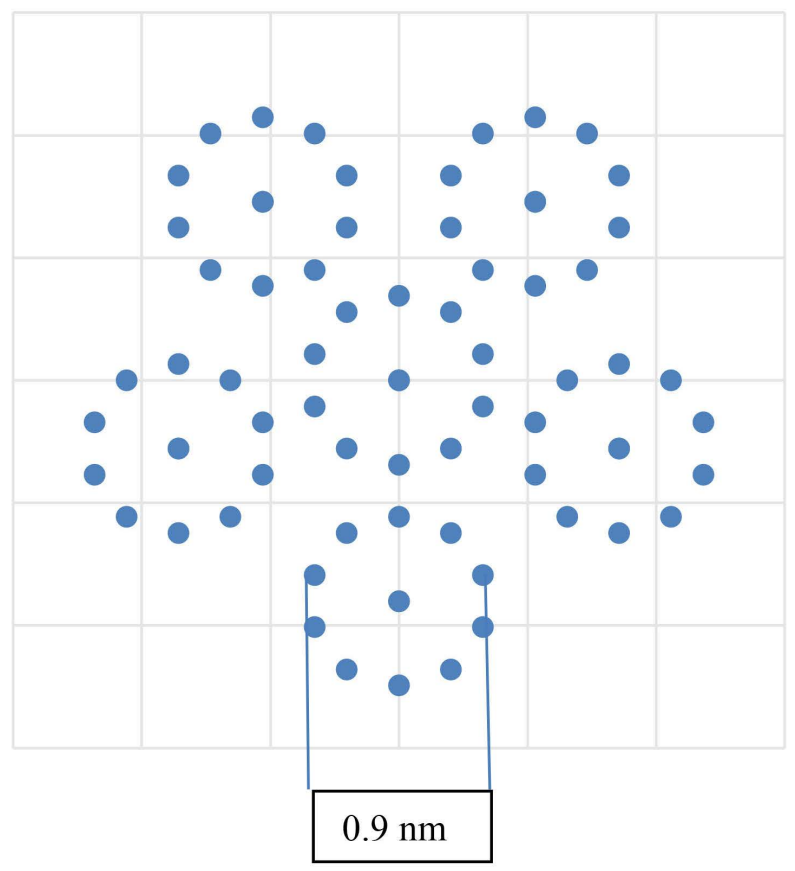

(a)

Supercluster order 2 , thickness $\tau^{2}$

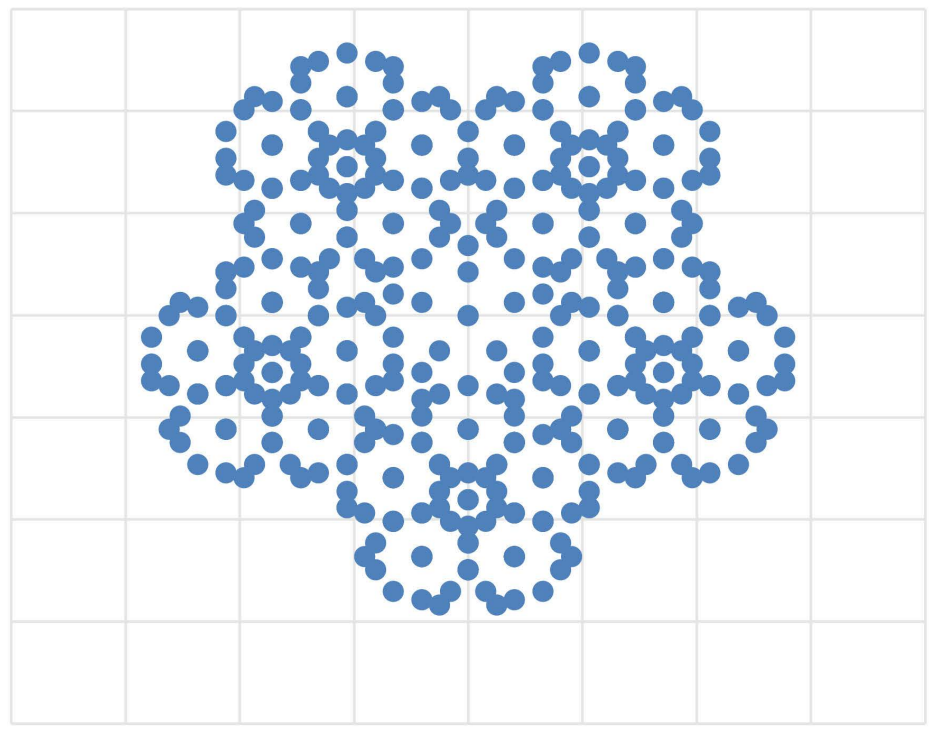

(c)

\section{Cluster peripheries in reverse contrast}

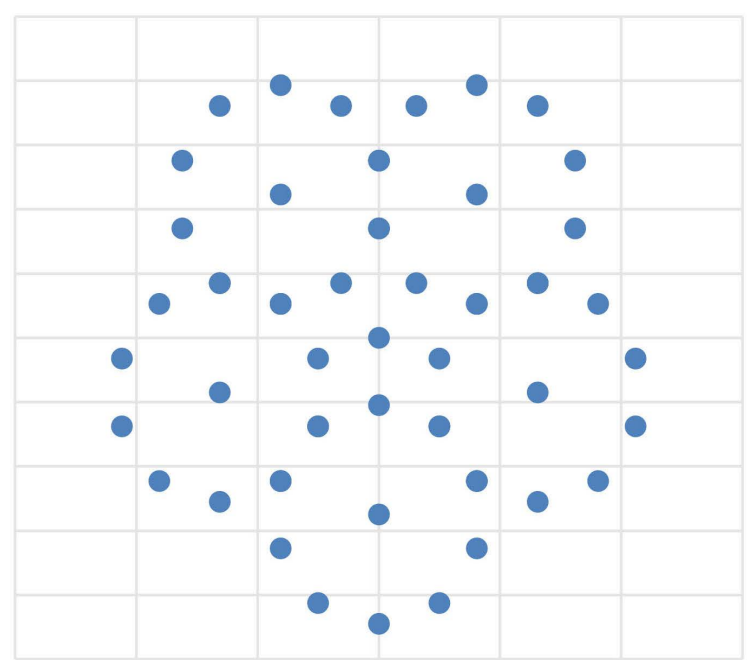

(b)

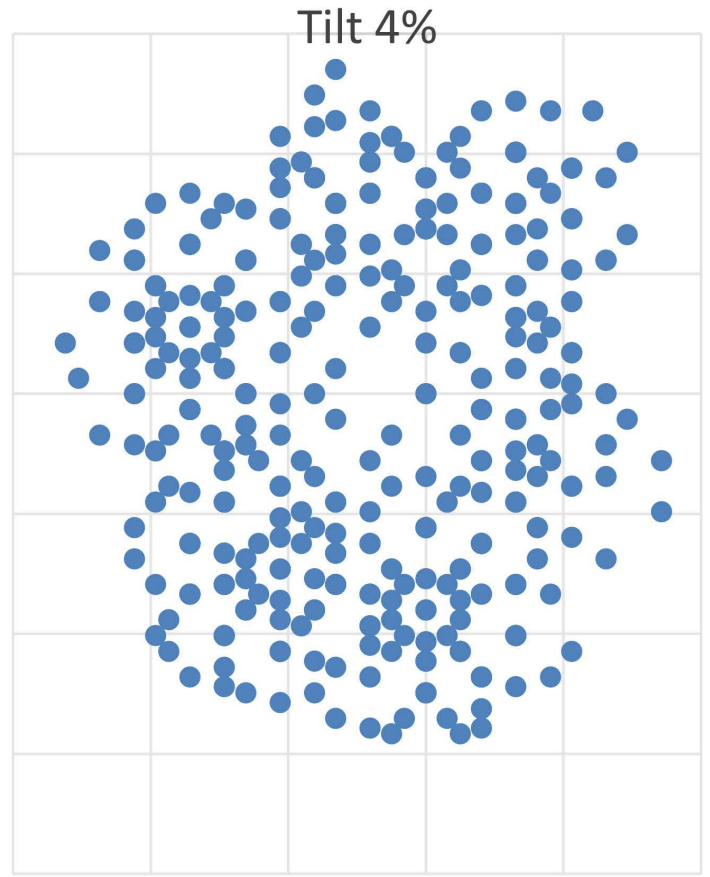

(d)

Figure 5. (a) Simulation of an HI supercluster of HI unit cell centers. The sample orientation is (1 $\tau 0)$ horizontal. The diameter of the circle of cell centers is $0.9 \mathrm{~nm}$. (b) Simulation in reverse contrast that matches the corresponding pattern in Bursill \& Peng [18] among others. (c) Simulation of a horizontal supercluster order 2. (d) Simulation of the same supercluster tilted about the vertical axis to a slope of 1:25. Notice that, even with small tilts, the pattern is lost except for apparently random cells and clusters. 
occur at a radius $\tau$ times greater than atomic separations from corresponding cluster centers. This process yields an excellent simulation of a supercluster in Bursill and Peng's image. In particular, the clusters in reverse contrast touch, i.e. at cluster edges $[5]^{6}$. Moreover, the dimensions are confirmed along with the hierarchic model up to four tiers of order. The match with Bursill and Peng becomes irresistible, and is generally comparable to other miscroscopic images of quasicrystals.

What about the supercluster order 2? This is shown in reverse contrast in Figure 5(c). As the images expand in area, they become harder to observe because of the precision required in locating a thin foil prepared by either electropolishing or ion milling. For example, an error of only 1:25 in the slope of the polished foil about the vertical axis yields the simulation shown in Figure 5(d). After tilting, the supercluster pattern is, for the most part, lost; though remnants of apparently random cells and clusters are identifiable. It is therefore impractical to expect that large areas of a QC should exhibit superclusters of high order. Nevertheless, hierarchic order is demonstrated in the images, along with their geometric series diffraction.

\section{Possibility for Quasi-spherical Cells}

In principle, the $\mathrm{Hi}$ is infinite in extension. The units are initially and progressively edge-sharing and holey. Defects have been a long-time concern, but given the firm ideal model, progressive solutions are possible.

Previously, we have considered defects in the short range: vacancies, interstitials, disloctions etc. They are natural products of the edge-sharing structure of the HI, but they are not sufficiently dense to noticeably affect the diffraction pattern. Now we consider defects in longer range.

Consider the regular icosahedron as being quasi-spherical. This is justified by its having 15 identical cross-sections (Figure 4) at various orientations. Secondly, consider metallic atoms as approximately spherical, so that they may, from a structural viewpoint, be replaced by icosahedra. We may therefore imagine two structures: one an fcc lattice with icosahedral unit cells; and another the icosahedral lattice containing fcc unit cells ${ }^{7}$. In neither case need the cells be edge sharing and space filling. What effect might transformations between the two structures have on the diffraction pattern, can be investigated by QSFs.

Simulated QSFs are shown in Figure 6. The specimen sizes are typical for a supercluster order 2. These are compared with each other, and also with the QSF calculated on an HI supercluster order 6, in Figure 2. It is clear that for pure fcc $\mathrm{Al}$ (Figure 6(a)) at the Bragg condition, $c_{s}=1$ at the origin, as it must be. In Figure 2, $c_{s}=0.894$ for $\mathrm{i}-\mathrm{Al}_{6} \mathrm{Mn}$, as described above.

${ }^{6}$ The simulation is partly fortuitous: the radius of the cluster is $\tau$ times the radus of the unit cell and coincides with the outer edge of cell, i.e. including both $\mathrm{Mn}$ and the outer lining of $\mathrm{Al}$ atoms.

${ }^{7}$ The fcc unit cell is cubic, but its stacking is similar to the unit cell in i- $\mathrm{Al}_{6} \mathrm{Mn}$ ([4], p.19). 


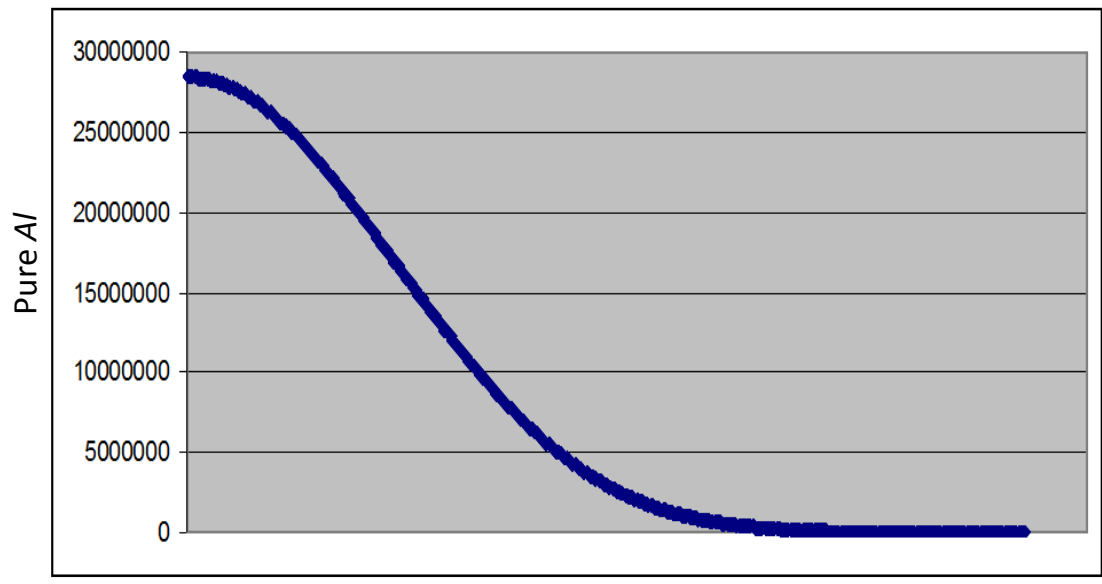

(a)

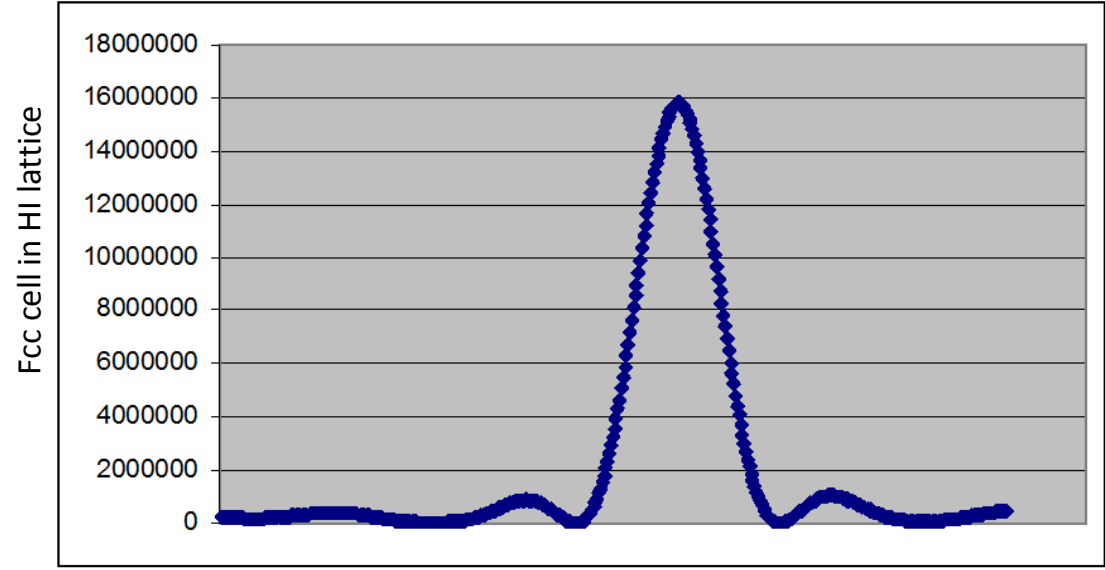

(b)

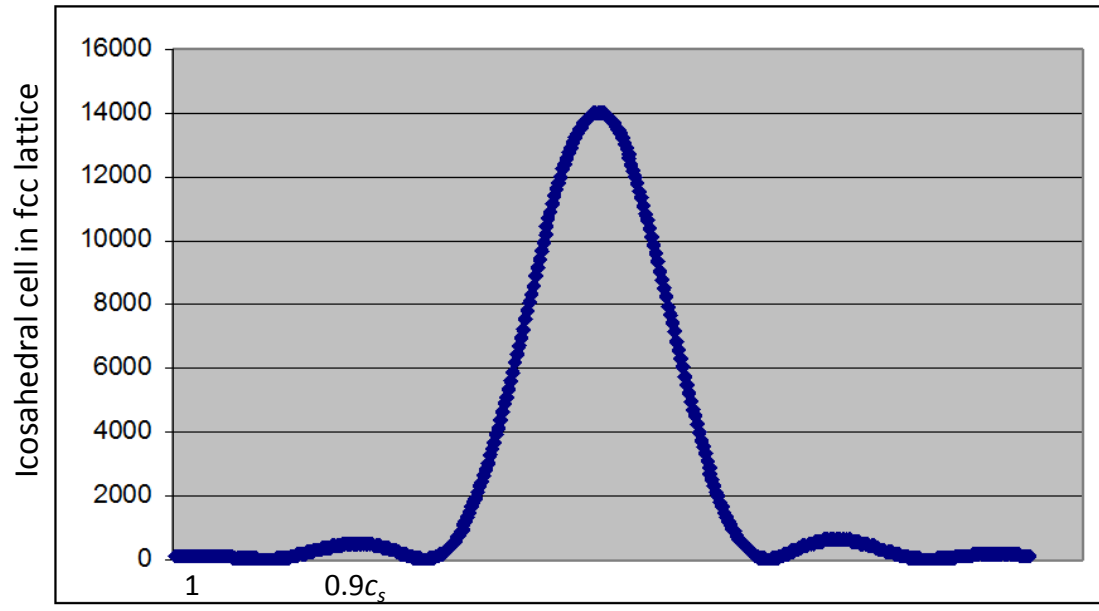

(c)

Figure 6. (a) QSFs for a cubic cluster of fcc Al, having about 20,000 atoms, similar to a supercluster order 2. Note $c_{s}=1$, i.e. Bragg reflection. (b) Computed QSF for (111) diffraction due to an imaginary fcc cell on an icosahedral grid, as in a supercluster order 2 . Note $c_{s} \simeq 0.9$. (c) Computed QSF for $(\tau, \tau, \tau)$ diffraction due to an icosahedral cell on an imaginary cubic grid of side $\tau$. Site population about 20,000 (like a and b). Notice that $c_{s}$ is similar to configuration in b. [Bourdillon, A.J. (2010) Quasicrystals' 2D Tiles in 3D Superclusters. UHRL, San Jose, ISBN 978-0-9789839-2-5 p. 66]. 
Meanwhile the two imaginary solids in Figure 6(b) and Figure 6(c) have $c_{s} \simeq$ 0.9. This simulation for the metrics of imaginary structures demonstrates that the QSFs are determined by the lattice as well as the unit cells. (contrast crystals, where SFs are calculated within individual cells and are identical for all cells and for the lattice). The observation implies that, if higher order icosahedra are substituted by cubic structures, or even by quasi-speherical structures, as defects in the quasi lattice, then the diffraction pattern may be approximated by the pattern that is calculated in the ideal HI model. The lattice may then be locally defective, while retaining the principal scattering properties of the ideal HI structure. By this means, a mixture of local quasi-cubic lattice sites with icosahedral quasi-lattice sites of high order might reduce density fluctuations. These would add to conventional vacancies and interstitials as possible defects in the long range. There is no evidence that this occurs, but the data signals that, with regard to long range defects, the geometric series diffraction in QCs is as robust as Bragg diffraction in crystals.

\section{Conclusions}

Scattering by Hiearchic Icosahedral structures is the most obvious instrument for diffraction of periodic probes into geometric series. The physical process for this fact has been described in detail. It is classical, 3-dimensional, and independent of tiling theory. The quantum requirements for the diffraction in geometric series contain necessary relationships of harmony and digitization. In consequence, resonant quasi-Bloch waves in the scattering probe have translational symmetry at geometric series orders $a \tau^{m}$. The Hierarchic Icosahedral structure is consistent with phase-contrast, optimum-defocus imaging. This is simulated by reverse-contrast mapping of atoms that scatter X-ray or electron probes incident on a thin QC foil. However, owing to the hierarchic translational symmetries, higher orders appear to randomize by specimen tilts of small angles away from horizontal. The difficulty of thin-film specimen preparation with optimized quasicrystollagraphic orientation results in the common conclusion: there is "no translational symmetry"; However this view not only contradicts expectations in "long range order", but is not necessary since hierarchic icosahedra indeed have translational symmetry. This occurs consistently, both in the hierarchic structure and in the resonant response.

Finally this solution for both the structure and diffraction differs from the two dominant objectives commonly followed in QC research: Our method is entirely classical and verified [2] [5] [6] [7]: it concentrates on the simplest and original (diatomic) system with minimal complication. The method has successfully identified the principal principles. Those other methods have been admittedly tentative and wishful for forty years. The first is mathematical and in-complete, viz. the mathematics of non-periodic tilings e.g. [19]; the second has covered for its shortcomings by spreading the net: the method attempts to find the extent of possible quasicrystals, especially with respect to composition and process e.g. 
[20]. The net has not uncovered the desired conclusion. Scientific method is empirical; we used to wish, and used to collect data, but our comprehension has since expanded to higher orders.

\section{Conflicts of Interest}

The author declares no conflicts of interest regarding the publication of this paper.

\section{References}

[1] Shechtman, D., Blech, I., Gratias, D. and Cahn, J.W. (1984) Physical Review Letters, 53, 1951-1953. https://doi.org/10.1103/PhysRevLett.53.1951

[2] Bourdillon, A.J. (2020) Journal of Modern Physics, 11, 581-592. https://doi.org/10.4236/jmp.2020.114038

[3] Bourdillon, A.J. (2011) Logarithmically Periodic Solids. Nova Science, New York.

[4] Bourdillon, A.J. (2012) Metric, Myth and Quasicrystals.

[5] Bourdillon, A.J. (2021) Journal of Modern Physics, 12, 1012-1026. https://doi.org/10.4236/jmp.2021.127063

[6] Bourdillon A.J. (2009) Solid State Communications, 149, 1221-1225. https://doi.org/10.1016/j.ssc.2009.04.032

[7] Bourdillon, A.J. (2017) Dispersion Dynamics in the Hall Effect and Pair Bonding in $\mathrm{HiT}_{\mathrm{c} .}$ Nova Science, New York.

[8] Hirsch, P., Howie, A., Nicholson, R.B., Pashley, D.W. and Whelan, M.J. (1977) Electron Microscopy of Thin Crystals. Krieger, New York.

[9] Bourdillon, A.J. (2010) Quasicrystals' 2D Tiles in 3D Superclusters. UHRL, San Jose.

[10] Bourdillon, A.J. (2018) Journal of Modern Physics, 9, 1304-1316. https://doi.org/10.4236/jmp.2018.96079

[11] Bourdillon, A.J. (2018) Journal of Modern Physics, 9, 2295-2307. https://doi.org/10.4236/jmp.2018.913145

[12] Bourdillon, A.J. (2020) Journal of Modern Physics, 11, 1926-1937. https://doi.org/10.4236/jmp.2020.1112121

[13] Bourdillon, A.J. (2013) Micron, 51, 21-25. https://doi.org/10.1016/j.micron.2013.06.004

[14] Bourdillon, A.J. (2009) Quasicrystals and Quasi Drivers.

[15] Bourdillon, A.J. (2020) Journal of Modern Physics, 5, 1079-1084. https://doi.org/10.4236/jmp.2014.512109

[16] Tsai, A.P. (2008) Science and Technology of Advanced Materials, 9, 1-20. https://doi.org/10.1088/1468-6996/9/1/013008

[17] Bourdillon, A.J. (1987) Philosophical Magazine Letters, 55, 21-26. https://doi.org/10.1080/09500838708210435

[18] Bursill, L.A. and Peng, J.L. (1985) Nature, 316, 50-51. https://doi.org/10.1038/316050a0

[19] Grimm, U. and Kramer, P. (2019) Quasicrystals.

[20] Steinhardt, P. (2018) The Second Kind of Impossible: The Extraordinary Quest for a New Form of Matter. Simon \& Schuster, New York. 